\section{POS0329 GASTROINTESTINAL INVOLVEMENT IN SYSTEMIC SCLEROSIS: PATHOGENETIC ROLE OF GUT MICROBIOME, CYTOKINES AND ADIPOKINES}

E. Pigatto ${ }^{1}$, M. Schiesaro2, M. Caputo ${ }^{3}$, M. Beggio ${ }^{3}$, P. Galozzi ${ }^{4}$, F. Cozzi2, P. Sfriso $0^{4} .{ }^{1}$ Fondazione Villa Salus, Medicine Unit, Venice, Italy; ${ }^{1}$ Fondazione Villa Salus, Medicine Unit, Venice, Italy; ${ }^{3}$ Fondazione Villa Salus, Laboratory Unit of clinical chemistry and microbiology, Venice, Italy; ${ }^{4}$ Azienda Ospedaliera di Padova, Rheumatology Unit, Padua, Italy

Background: Gastrointestinal (Gl) involvement is very common in patients with Systemic Sclerosis (SSc). The pathophysiology of GI manifestations has not yet been defined. Cell-mediated immunological reactions appear to lead to endothelial damage resulting in fibrosis. The risk of developing malnutrition reinforces the need to better understand GI pathophysiology in these patients.

Objectives: The study aimed to evaluate GI symptoms (GIT 2.0) and malnutrition status (MUST) and to determine specific bacterial changes in gut microbiome by investigating the possible presence of positive hot spots in bacterial species in SSc patients and their potential role in the disease progression. We also evaluated serum levels of adipokines and cytokines involved in the pathogenesis of SSc and their role, in addition to gut microbiome, in predicting the onset of Gl involvement and malnutrition in SSc patients.

Methods: We enrolled 25 scleroderma patients (EULAR/ACR 2013 criteria). UCLA-SCTC GIT 2.0 questionnaire to evaluate GI symptoms and MUST to investigate the risk of malnutrition were used. Gut microbiome was analyzed and the samples were subjected to extraction for the 16S rRNA gene (Earth Microbiome Project and the NIH-Human Microbiome Project). The microbiome was investigated at phenotypic and genotypic level. Serum levels of cytokines and adipokines (adiponectin and leptin) were evaluated by ELISA.

Results: $79.9 \%$ of patients had GERD and $63.5 \%$ abdominal distension at GIT 2.0 questionnaires. $48 \%$ of patients had moderate risk of malnutrition (MUST=2) and $12 \%$ had high risk (MUST=3). Gut microbioma: 19 patients $(76 \%)$ had low similarity and $11(44 \%)$ low diversity compared to the healthy population. The prevailing enterotypes of gut microbiome was Bacteroides (80\%) and Prevotella $(20 \%)$. The genotypic evaluation showed a reduced concentration of: gluten-digesting (Lactobacillus); lactose-digesting (Faecalibacterium); vitamin K-producing (Enterococcus, Desulfovibrio and Veillonella); acetaldehyde-degrading bacteria. 24 patients (96\%) showed a reduction in bacteria devoted to maintaining weight control (Bifidobacterium and Ruminococcus). The patients had an altered intestinal permeability with less mucolytic bacteria (Bacteroides) and reduced production of LPS (Enterobacter and Escherichia). Low levels of butyrate (Eubacterium and Clostridium), acetate and propionate were found for SCFA-producing bacteria. Potentially pathogenic bacteria were also investigated: Salmonella was found in $14(56 \%)$, Klebsiella in $9(36 \%)$ and Enterococcus Faecalis in $3(12 \%)$ patients. $11(44 \%)$ patients had elevated serum levels of IL10 and IL12; $4(16 \%)$ had high value of leptin. Correlation was found in patients who had a reduced concentration of gluten-digesting bacteria and MUST. Elevated MUST was correlated with serological increase in IL17A and IFN- $\alpha$. Serum levels of IL12 and IL10 were found to correlate with specific bacteria alterations: high concentration of acetaldehyde-producing bacteria and low levels of acetaldehyde-degrade bacteria (also correlated with high serum levels of IL6), mucolytic bacteria and producers of hydrogen sulphide, acetate and propionate. Finally, reduced levels of mucolytic bacteria and acetate producing bacteria correlated with high serum leptin levels.

Conclusion: The relationship between the gut microbiome and SSc seems to be multifactorial. In our study genotypic changes of gut microbioma might play a role in damaging the permeability of the mucosa and increasing risk of malnutrition. The evaluation of gut microbiome and cytokine profile is probably going to be of value in the follow-up of SSc. However, further studies are needed to clarify the impact of Gl dysbiosis on the immune system in SSc.

REFERENCES:

[1] Patrone V. et al. Gut microbiota profile in systemic sclerosis patients with and without clinical evidence of gastrointestinal involvement, Sci Rep. 2017; 7: 14874

Disclosure of Interests: None declared

DOI: 10.1136/annrheumdis-2021-eular.1904

\section{POS0330 NINTEDANIB (TYROSINE KINASE INHIBITOR) DOWNREGULATES THE TRANSITION OF CULTURED SYSTEMIC SCLEROSIS FIBROCYTES INTO MYOFIBROBLASTS AND THEIR PRO-FIBROTIC ACTIVITY}

S. Soldano $^{1}$, P. Montagna ${ }^{1}$, E. Gotelli ${ }^{1}$, S. Tardito ${ }^{1}$, S. Paolino ${ }^{1}$, C. Corallo ${ }^{2}$, C. Pizzorni ${ }^{1}$, A. Sulli ${ }^{1}$, C. Schenone ${ }^{1}$, G. Pacini ${ }^{1}$, V. Smith ${ }^{3,4,5}$, M. Cutolo $^{1}$. ${ }^{1}$ University of Genova, IRCCS San Martino Polyclinic Hospital, 1 Laboratory of
Experimental Rheumatology and Academic Division of Clinical Rheumatology, Dept. Internal Medicine, Genoa, Italy; ${ }^{2}$ University of Siena, 2 Scleroderma Unit, Department of Medicine, Surgery and Neurosciences, Siena, Italy; ${ }^{3}$ Ghent University Hospital, Ghent, Department of Rheumatology, Ghent, Belgium ${ }^{4}$ Ghent University, Department of Internal Medicine, Ghent, Belgium; ${ }^{5} \mathrm{VIB}$ Inflammation Research Center (IRC), Unit for Molecular Immunology and Inflammation, Ghent, Belgium

Background: Fibroblast-to-myofibroblast transition is one of the fundamental steps involved in the fibrotic process that characterise systemic sclerosis (SSc) [1]. Myofibroblasts are $\alpha$-smooth muscle actin ( $\alpha \mathrm{SMA}$ ) positive cells that contribute to fibrosis through the excessive synthesis and deposition of extracellular matrix (ECM) proteins, primarily fibronectin (FN) and type I collagen (COL1) [2] Among the cells involved in the fibrotic process of SSc, circulating fibrocytes seem to have an emerging role as an important source of fibroblasts and myofibroblasts [3].

Nintedanib is a tyrosine kinase inhibitor approved for the treatment of idiopathic pulmonary fibrosis that interferes with the signalling pathways involved in the pathogenesis of fibrosis (4). Nintedanib was recently demonstrated to have a beneficial effect in patients with interstitial lung disease (ILD) associated with SSc (5).

Objectives: To investigate nintedanib effect in inhibiting the in vitro transition of circulating SSc fibrocytes into myofibroblasts and their pro-fibrotic activity.

Methods: Circulating fibrocytes were obtained from 14 SSc patients (mean age $64 \pm 14$ years), who fulfilled the 2013 ACR/EULAR criteria for SSc and that underwent complete disease staging in a day-hospital setting at the Rheumatology Division of Genoa University. Five age-matched healthy subjects (HSs) were also analysed. All SSc patients and HSs signed the informed consent and the local EC approved the study. Peripheral blood mononuclear cells were isolated by density gradient centrifugation and plated on $\mathrm{FN}$-coated dishes. After overnigh culture, non-adherent cells were removed, and adherent cells were maintained in growth medium for 8 days (T8) to obtain fibrocytes [6]. T8-cultured SSc fibrocytes were maintained in growth medium (untreated cells) or treated with nintedanib $0.1 \mu \mathrm{M}$ and $1 \mu \mathrm{M}$ for 3 and 24 hours. Fibroblast specific protein-1 (S100A4) and aSMA, as markers of fibroblast/myofibroblast phenotype, together with COL1 and FN, were investigated by qRT-PCR and Western blotting. Non-parametric Mann-Whitney and Wilcoxon tests were used for the statistical analysis.

Results: Significantly elevated gene and protein expressions of aSMA, S100A4 COL1 and FN were observed in SSc fibrocytes compared to HS fibrocytes (gene: $\alpha$ SMA $p<0.001$; others $p<0.0001$; protein: all $p<0.05$ ). In accordance with the antibody positivity for Scl70 and the presence or absence of ILD at CT scan, SSc patients were grouped as either Scl70 positive patients with ILD (Scl70 $\left.{ }^{+} \mid L^{+}\right)$ or Scl70 negative patients without ILD (Scl70'ILD'). Significant $\alpha$ SMA, S100A4 COL1 and FN gene expressions were found in fibrocytes from Scl70 ILD compared to HS fibrocytes ( $\alpha$ SMA $p<0.001$; others $p<0.0001$ ). Moreover, fibrocytes from Scl70+ILD+patients showed a more significant gene expression of fibroblasts/myofibroblasts markers compared to Scl70'ILD patients $(p<0.01$ for S100A4), whereas no differences were observed for ECM gene expression. Nintedanib reduced the gene and protein expression of $\alpha \mathrm{SMA}, \mathrm{COL} 1$ and FN in SSc fibrocytes compared to untreated ones with different statistical significance. Noteworthy, nintedanib significantly downregulated $\alpha$ SMA, S100A4, COL1 and FN gene expression (all $\mathrm{p}<0.05$ ) in $\mathrm{Scl} 70^{+} \mid \mathrm{LD}^{+}$fibrocytes, whereas only that of S100A4 and FN was significantly downregulated $(p<0.05)$ in Scl70'ILD' fibrocytes compared to untreated cells.

Conclusion: Nintedanib seems to downregulate in vitro the transition of fibrocytes into myofibroblasts and their pro-fibrotic activity, particularly in cells isolated from Scl70+ILD+SSc patients.

\section{REFERENCES:}

[1] Cutolo M et al. Exp Rev Clin Immunol. 2019;15:753-64.

[2] Van Caam A et al. Front. Immunol. 2018;9:2452.doi:10.3389/ fimmu.2018.02452.

[3] Distler JH et al. Arthritis Rheumatol. 2017;69:257-67.

[4] Distler O et al. New Eng J Med. 2019; 380:2518-28.

[5] Maher TB et al. Arthritis Rheumatol.2020.doi:10.1002/art.41576.

[6] Cutolo Metal.Arthritis Res Ther.2018;20:157.doi:10.1186/s13075-018-1652-6. Acknowledgements: We thank Stefano-Lutz Willing for the scientific support through the study.

Disclosure of Interests: Stefano Soldano: None declared, Paola Montagna: None declared, Emanuele Gotelli: None declared, Samuele Tardito: None declared, Sabrina Paolino: None declared, Claudio Corallo: None declared, Carmen Pizzorni: None declared, Alberto Sulli: None declared, Carlotta Schenone: None declared, Greta Pacini: None declared, Vanessa Smith: None declared, Maurizio Cutolo Grant/research support from: I received grant/research support from Bristol-Myers Squibb, Boehringer, Celgene DOI: 10.1136/annrheumdis-2021-eular.2411 